\title{
Studying Efficiency of NDE Techniques Applied to Composite Materials in Aerospace Applications
}

\begin{abstract}
K. DRAGAN ${ }^{a}$ AND W. SWIDERSKI ${ }^{b}$
${ }^{a}$ Air Force Institute of Technology, Księcia Bolesława 6, 01-494 Warsaw, Poland

${ }^{b}$ Military Institute of Armament Technology, Prymasa Wyszyńskiego 7, 05-220 Zielonka, Poland

This work presents the modern approach to the detection of various types of defects in composite structures used in aerospace. In such structures, including glass reinforced plastics and carbon reinforced plastics, different failure modes could occur at a manufacturing stage and during service life. Defects are connected with inadequate technology, poor workmanship, cycling fatigue loads, impact damage and environmental conditions. The main types of defects are delaminations, disbonds, foreign object inclusions and porosity. To detect such defects, several non destructive evaluation techniques can be applied, merely to mention ultrasonic, low frequency acoustics, infrared thermography and shearography. The use of multimode non destructive evaluation techniques enables characterization of defects which cannot be detected by using single non destructive evaluation methods. This paper demonstrates the necessity of using non destructive evaluation methods for the implementation of quality control and maintenance procedures while servicing aerospace composite elements.
\end{abstract}

PACS numbers: 81.70.Cv, 07.57.-c, 62.20.M, 07.05.Pj, 62.20.mt

\section{Introduction}

Composite structures are becoming increasingly popular in the aerospace industry due to their unique features, such as excellent strength/weight ratio, corrosion resistance and the possibility to manufacture samples of complicated shapes. Nowadays, the extensive use of composites in Aerospace components has become a real fact; for example, in the Boeing 787 Dreamliner aircraft, nearly $50 \%$ of structures have been made of composite materials [1].

Presently, such responsible components as main rotor blades (MRB) of helicopters are also manufactured of composite materials. This study is devoted to the study of aerospace composite elements, such as epoxy and carbon epoxy parts. These parts are characterized by a laminar and sandwich structure. There is a number of failure problems which may appear in composites being related to both a manufacturing and operating stages. The typical failures, which affect structural integrity and residual strength of the composites, include disbonds, delaminations, foreign object inclusions and a kind of damage which is called barely visible impact damage [2-4]. Characterization of such failure modes is not easy when using single nondestructive testing (NDT) techniques [5]. In order to perform a complete damage description, multimode non destructive evaluation (NDE) of composites should be implemented. A list of candidate techniques may include ultrasonic, mechanical impedance analysis (shearography), thermography, X-ray radiography and some others.

\section{Inspection approach}

Depending on the inspection technique, several physical phenomena can be considered to achieve the inspec- tion goal, i.e. to evaluate a type of failure in materials under test.

These phenomena are connected to both a physical nature of excitation signals and a type of inspected materials. Attenuation of acoustic waves in composites depends on the thermal conductivity of fibers (glass or carbon), type of structure (sandwich or laminar), as well as material quality. The analysis of the corresponding physical phenomena is of a primary interest [6] because they decide about a proper choice of a NDE technique [7], especially if to take into account that the number of failure scenarios is vast [8]. The most expected failure modes have been studied in this work along with the corresponding experimental results.

\subsection{Detecting disbonds}

Adhesive joints are commonly used in the manufacturing of aerospace components (the so-called bondlines). In particular cases, this bonding technique is supposed to be more efficient than regular ones, such as bolts, rivets etc. Moreover, it allows for the integration of different materials (composite-to-metal, skin-to-honeycomb, etc.), as well as the conduction of a possible future repair. However, such joints may collapse because of the presence of manufacturing faults, operating cyclic loads and impact damages. Depending on a particular bondline and a type of structure, the inspection of adhesive joints may be accompanied by some problems. At the maintenance stage, because of a specific stress distribution and operating loading cycles, critical areas may propagate from bondline edges, as shown in Fig. 1.

To apply NDE techniques for the inspection of disbonds, some standard specimens, used in Ultra-Light aircraft, as well as in gliders and rotor blades of helicopters, were manufactured. The first sample was the rudder of 


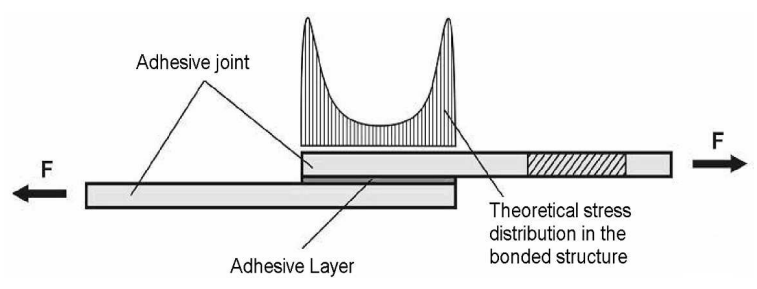

Fig. 1. Stress distribution in the bondline.

a Poland-made glider PW-5 (Fig. 2).

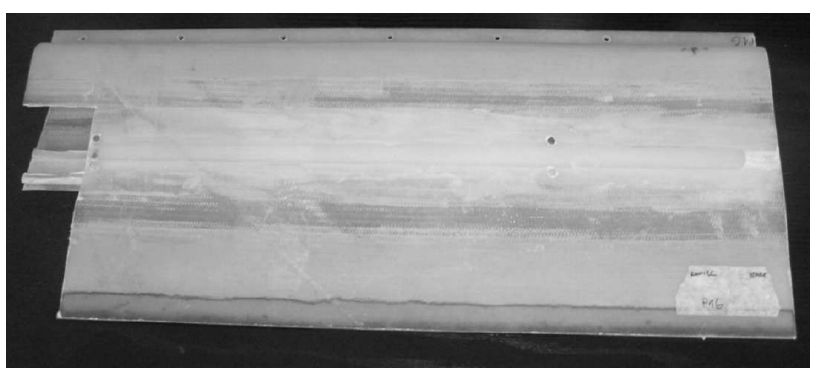

Fig. 2. Rudder of the PW-5 glider.

The start inspection technique used to monitor bond quality was ultrasonic [9]. This technique enables the generation of elastic waves in structures under test. The following formula describes the propagation of elastic waves [10]:

$$
(\lambda+\mu) u_{j, j i}+\mu u_{j, j j}+\rho f_{i}=\rho \ddot{u} .
$$

Here $\lambda$ and $\mu$ are the Lame constants, $\rho$ is the medium density, and $u$ is the displacement. In the case of isotropic media, Eq. (1) allows to obtain solutions for different coordinate directions. Anisotropic composites represent a more difficult case because their acoustic properties vary with fiber directions. There are some special inspection devices which allow to evaluate both propagation direction and signal amplitudes. In composites, the attenuation of acoustic waves, which mainly appears due to scattering and reflection, is an important phenomenon which limits wave propagation. Scattering is reduced to minimum if the acoustic impedance of the fibers $Z_{\mathrm{z}}$ is close to the acoustic impedance of the matrix $Z_{\mathrm{o}}$ [11]:

$$
Z_{\mathrm{z}} \cong Z_{\mathrm{O}},
$$

with the length of the acoustic wave meeting the condition

$$
l \gg d \text {. }
$$

Here $l$ is the acoustic wavelength, and $d$ is the fiber diameter.

In practice, the reduction of scattering is effectively achieved when:

$$
l_{\min }=a d,
$$

where $a$ is the coefficient of which value is in the range from (3) to (4). The last equation allows the empirical determination of acoustic wave frequency:

$$
f=c /(a d),
$$

where $c$ is the wave velocity. Choosing a correct frequency requires a certain compromise between the sensitivity (a lower frequency reduces sensitivity) and the attenuation which increases with higher frequencies. Another issue is related to material thickness. The example presented in Fig. 1 shows a very thin skin attached to the reinforcement through an adhesive. The corresponding ultrasonic C-scan is shown in Fig. 3.

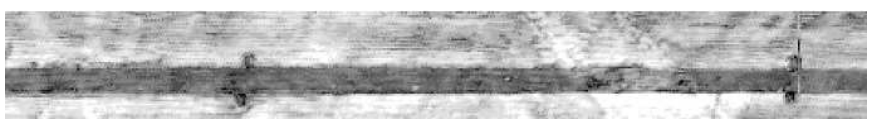

Fig. 3. Ultrasonic C-scan of the PW-5 rudder.

The bondline of the joint is clearly seen in this scan image. The ultrasonic assessment of bondlines is possible by using a $6 \mathrm{~dB}$ criterion or another signal index, such as signal-to-noise ratio (SNR) [12]:

$$
\mathrm{SNR}=\sqrt{\frac{16}{\rho V W_{x} W_{y} \Delta t}} \frac{A_{\mathrm{f}}\left(f_{\mathrm{o}}\right)}{\mathrm{SN}\left(f_{\mathrm{o}}\right)},
$$

where $\rho$ is the material density, $V$ is the sound speed in the material, $W_{x}$ and $W_{y}$ are the lateral beam widths, $A_{\mathrm{f}}$ is the amplitude signal from the flaw (at a centre frequency) and $\mathrm{SN}$ is noise at signal center frequency. The use of the criterion above enables the bondline evaluation

The next inspection NDE technique has been infrared (IR) thermography, which can be implemented in both passive and active modes. The passive IR thermographic testing is applied to objects of whose temperature is different from the ambient one, whether it is due to natural or exploitation reasons. Typically, evaluation of material defects is possible in the active mode where transient thermal fluxes are stimulated in samples under test by means of external heat sources [18, 19]. Several inspection techniques have been developed, merely to mention pulsed thermography, step heating, lock-in thermography, vibrothermography, etc. In all these procedures, either a reflection or transmission inspection procedures can be implemented. In the reflection procedure, a thermal source and an IR camera are located on the same side of a tested object, while in the transmission procedure two opposite object surfaces are respectively heated and monitored. In the IR thermogram of Fig. 4 (pulsed thermography, reflection method), the quality of the rudder glue joint can be easily evaluated.

Another inspection solution was X-ray radiography. Its advantage is the direct imaging of a sample internal geometry. In Fig. 5, both the bondline and the missing part of the internal rib are seen. The disadvantages of $\mathrm{X}$-ray radiography are the necessity to access both sides of samples and insensibility to certain types of defects, e.g. delaminations [13].

Also, the so-called kissing bonds can be hardly evaluated by using the ultrasonic technique [14]. To detect such defects, other NDE techniques should be used, such 


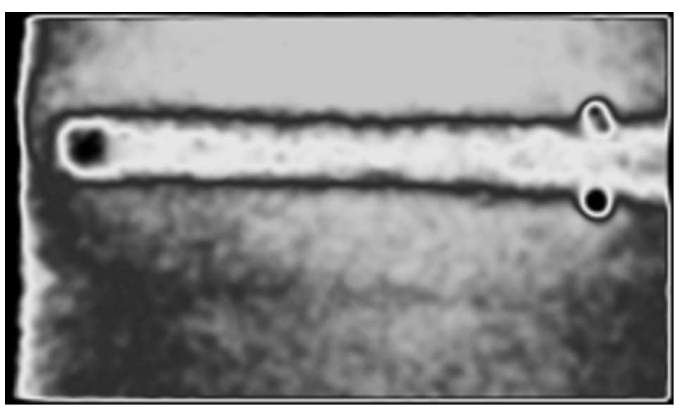

Fig. 4. IR thermogram of the PW-5 rudder.

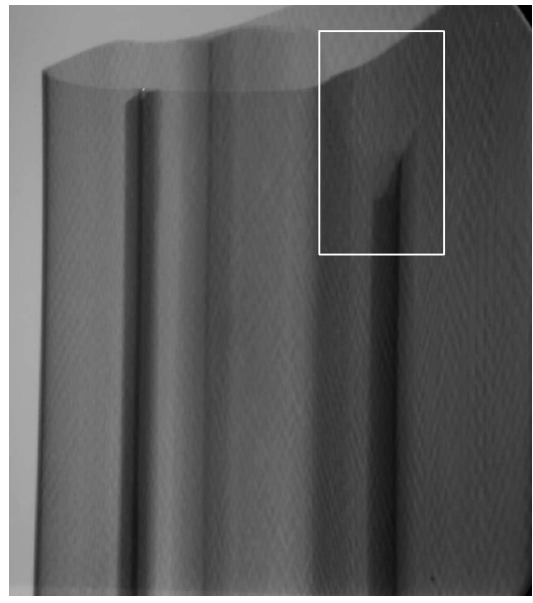

Fig. 5. Röntgenogram of the PW-5 rudder.

as mechanical impedance analysis and shearography [2].

The next studied parts represented thicker aircraft structures (e.g. wing skin — see Fig. 6) adhesively joined to substructure (spar, rib).

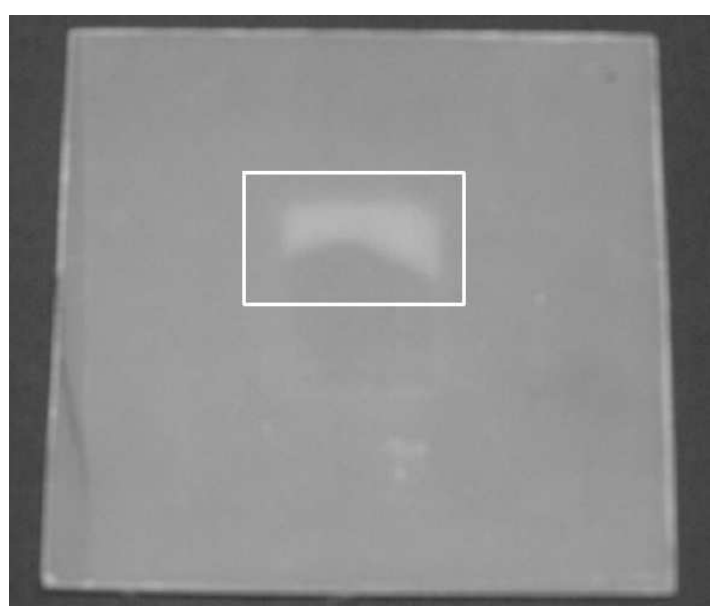

Fig. 6. Wing skin sample with a disbond.

The use of ultrasonic NDE for the inspection of thicker composites may be difficult because of problems related to attenuation of ultrasonic waves. To characterize this phenomenon, some techniques of attenuation measurement can be used. These techniques are based on the measurement of [15]: overall damping or ultrasonic attenuation (double through transmission) (dB); damping or ultrasonic attenuation per $\mathrm{mm}(\mathrm{dB} / \mathrm{mm})$ with the use of pulse echo. As always, a compromise between the frequency and the sensitivity should be achieved when inspecting thick or high-dispersive composites.

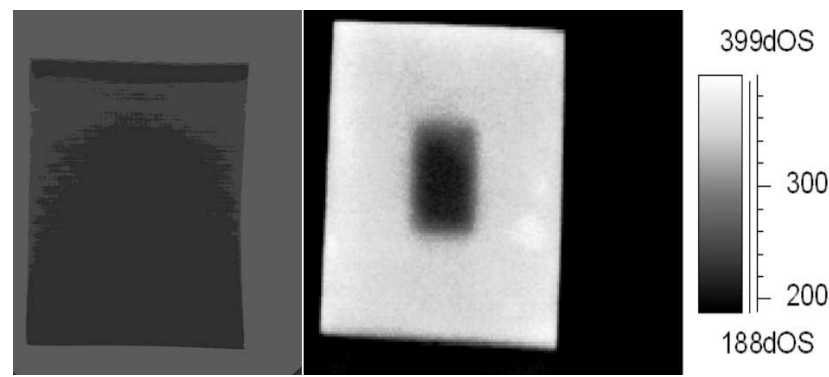

Fig. 7. Wing skin sample with a disbond results collected by using (a) X-ray radiography; (b) IR thermography.

Figure 7 demonstrates the images collected by using $\mathrm{X}$-ray radiography (a) and IR thermography (b) lock-in technique, phasegram.

The disbond in this sample can be well-detected with the ultrasonics. For thicker structures, the focused ultrasonic, or phased array technique, can be applied in order to increase the power spectrum of ultrasonic waves introduced into material.

In many aerospace structures, particularly used in small-size airplanes, sandwich panels stiffened with foam-to-core material are frequently used. In such panels, continuing loading and specific stress distribution (see Fig. 1) may cause disbonds between composite layers and cores. The corresponding sample was produced by joining a glass epoxy and a foam core. Disbonds of different size and shape were introduced into the sample to allow for the evaluation of testing efficiency.

Examination of such structures is difficult, especially by using the ultrasonic technique because of a low mechanical impedance of a core that may be expressed as follows:

$$
Z_{i}=\rho_{i} c_{l}^{(i)} \approx \sqrt{\rho_{i} E_{i}} .
$$

Low mechanical impedance is caused by low density of a core material. The use of a single ultrasonic sensor in the framework of the pulse-echo technique has proven to be inefficient. To improve the detection efficiency, ultrasonic phased arrays have been used. Successful experimental results in the inspection of the above-mentioned structure were obtained by focusing the beam and defining the aperture.

Figures 9a,b exhibits the data collected by using an ultrasonic phased array system. The visualization technique based on colours enabled the evaluation of defect depth, as well as the size and shape description. 


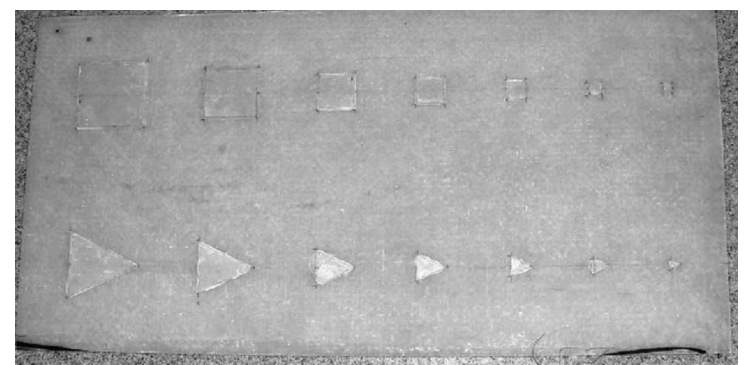

Fig. 8. Bondline between glass epoxy and foam core.

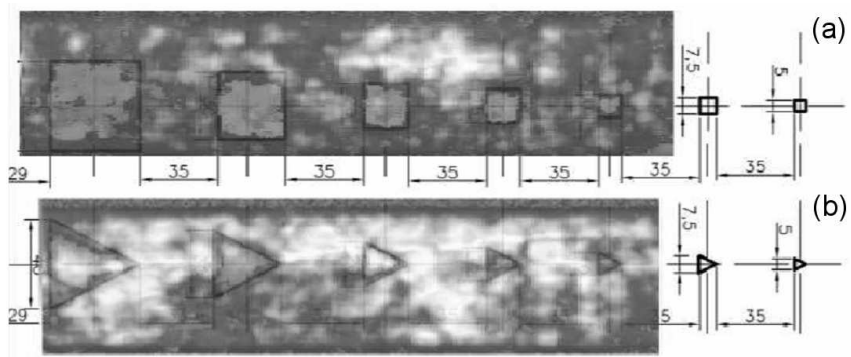

Fig. 9. Ultrasonic inspection results. (a) top and (b) bottom of sample from Fig. 8 .

The same sample was also checked thermographically to demonstrate a good visibility of all defects.

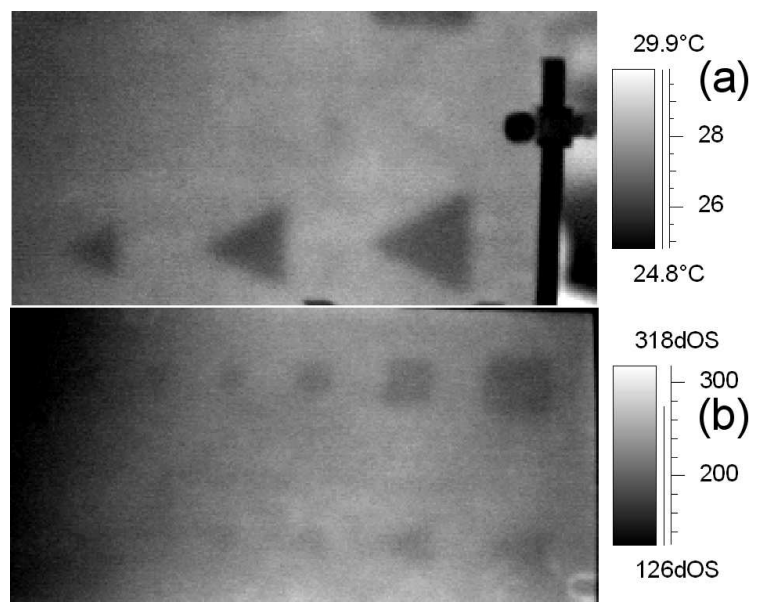

Fig. 10. Thermography results; (a) results of the IR thermogram; (b) results of lock-in IR thermography technique.

The corresponding results are presented in Fig. 10. The top image (a) shows the IR thermogram of the sample from Fig. 8 obtained in the transmission mode. The sample phasegram obtained by applying the lockin IR thermography technique is shown on the bottom (Fig. 10b).

\subsection{Detecting delaminations and inclusions}

During the manufacturing, some structural elements, such as peel plies, paper etc., may not be properly removed from a composite. In a final product, the presence of such elements, called foreign inclusions, affects mechanical properties (durability) of composites. Inclusions may not be visually seen, particularly, in carbon and/or painted (opaque) parts, but they can be effectively detected, for instance, ultrasonically.

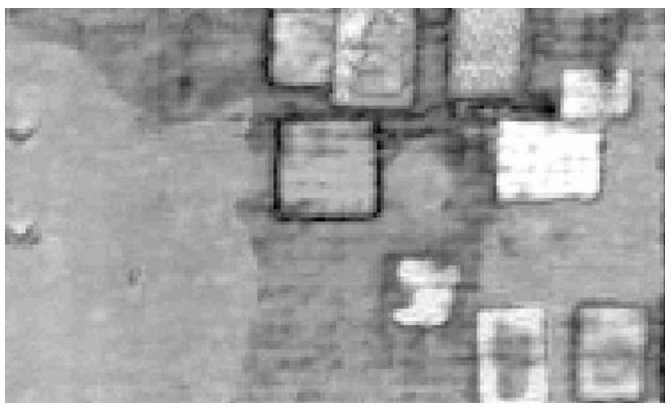

Fig. 11. Ultrasonic inspection result.

Figure 11 shows the result of the ultrasonic detection of foreign inclusions and delaminations in a laminar carbon fiber composite. All defects are clearly seen by applying the pulse-echo ultrasonic technique. Assessment of defect size and depth is also possible while analyzing ultrasonic data.

The IR thermographic inspection of the same sample resulted in the detection of maximum 7 defects from actually existing 9 defects, depending on data processing technique applied.

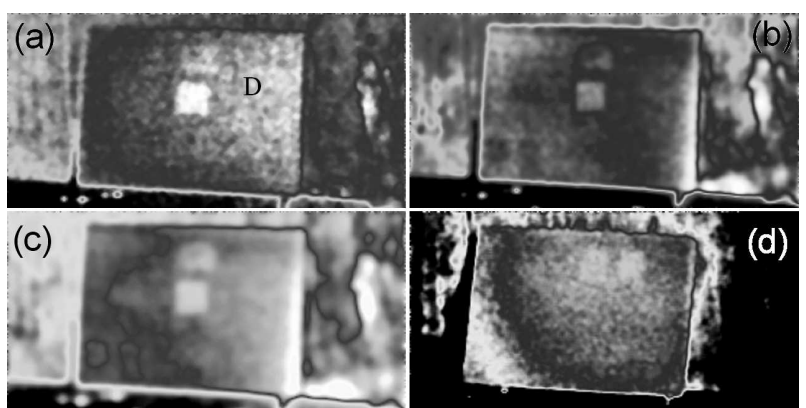

Fig. 12. IR thermograhy inspection results. (a) transmission procedure - the optimum source image, (b) PCA - the image of the 4th principal (c) PCA - the image of the 2nd principal, (d) reflection procedure the optimum source image.

Both reflection and transmission testing modes have been analyzed. Figure 12 shows the results obtained by applying principal component analysis (PCA). The SNR value between a particular defect area $D$ in Fig. 12a (thermogram, transmission procedure) and a neighbor non-defect area has been chosen to compare quality of thermograms. The optimum source image (see Fig. 12a) is characterized by $\mathrm{SNR}=6.7$. The maximum value of $\mathrm{SNR}=8.5$ has been observed in the image of the 2 nd 
principal component (PCA algorithm - see Fig. 12c). The image in Fig. 12b shows the 4th principal component $(\mathrm{SNR}=3.5)$, while Fig. $12 \mathrm{~d}$ corresponds to the reflection mode.

In the application to the same sample, X-ray radiography has produced no positive result because delaminations do not change an overall material; material density [13], even if radiation energy has been chosen properly.

One more type of serious defects which might appear in composites is impact damage which affects the structural integrity of components [16]. According to R. Smith [17], Composite structures can suffer quite severe impact damage without a noticeable surface indentation - known as barely — visible impact damage (BVID) — and this makes large - area defect detection a necessity for critical structural components. Impact damages may appear at both manufacturing and in-service stages if solid bodies, such as hail, stones, bullets etc., strike component surface. Low-energy damage is often accompanied by no visible surface indications but may significantly worsen the component structural integrity [16] by producing delaminations, disbonds, multiple cracks etc. In complex structures, such as rotor blades, structural defects can be classified by the following groups:

— skin defects (delaminations, cracks);

— skin to honeycomb defects (disbonds);

- honeycomb defects (core crush).

Figure 13 shows disbonds and delaminations detected with the use of mechanical impedance analysis which were produced by the impact of the so-called blunt impactor [2]. Since impact damage affects residual strength of composite parts, its duly detection represents an important diagnostic problem. One of the techniques potentially used for inspection of disbonds and delamination is shearography. The technique is based on the deformation gradient detection and the inspection time is much shorter in comparison to ultrasonic or mechanical impedance analysis.
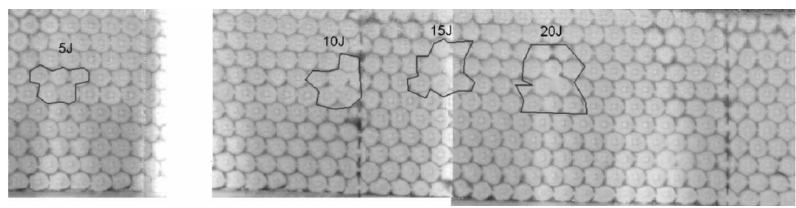

Fig. 13. Disbonds and delaminations detected with the mechanical impedance analysis.

If we define transformation direction parallel to the $x$ axis, the equation which describes the phase relation may be presented as follows [20]:

$$
\Delta \varphi=\frac{2 \pi}{l}\left(A \frac{\partial u}{\partial x}+B \frac{\partial v}{\partial x}+C \frac{\partial w}{\partial x}\right) \Delta x
$$

where
$\Delta \varphi$ - phase change of the collected signal for the two neighbor points on the surface under the deformation;

$l$ - wavelength;

$u, v, w$ - displacement vector (for the $x$ direction);

$\Delta x$ - shear vector for the $(x, y)$ plane;

$A, B, C$ - constant parameters.

In figure Fig. 14, shearography results are presented for the skin to honeycomb disbonds caused by low energy impact.

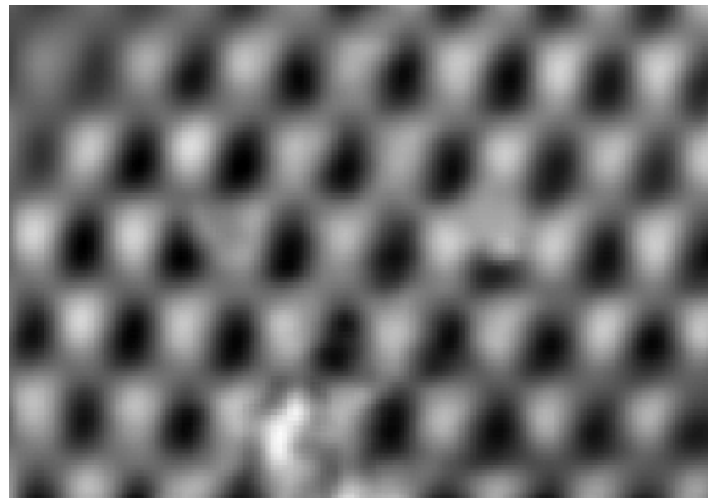

Fig. 14. Skin to honeycomb disbonds with shearography.

\section{Summary}

This paper describes some diagnostic problems related to the assessment of structural integrity of composites. There is a variety of defects which may appear in composites. Some of them, such as disbonds and delaminations, are quite typical, while others (porosity, inclusions) appear randomly, in particular, in the manufacturing process. Very crucial for composites is impact damage which may produce the so-called Barely Visible Impact Damage. Often, impact damage is accompanied by no visible surface marks however, it might significantly affects residual strength of materials.

In the aerospace industry, there is a bunch of NDE solutions intended for characterization of composite integrity. A type of materials, component design and peculiarities of access to inspected components decide about choosing a proper NDE techniques. In this study, some approaches to the detection of disbonds, delaminations and foreign object inclusions have been analyzed. In particular, three NDE techniques have been explored, namely, ultrasonics, IR thermography and X-ray radiography. The limits of the ultrasonic method are given by attenuation of elastic waves in particular materials; also, this technique cannot be used for the detection of kissing bonds. IR thermography works well in the case of disbonds, however, the efficiency of this technique is limited by a type of materials under test and availability of proper heat sources. We believe that optical flash and recently-appeared ultrasonic types of thermal stimulation produce the most promising results. 
$\mathrm{X}$-ray radiography is often useless in the detection of delaminations, and this technique requires access to both sides of tested samples, that might be no case when inspecting aerospace components.

\section{References}

[1] D. Roach, J. DiMambro, Enhanced Inspection Methods to Characterize Bonded Joints: Moving Beyond Flaw Detection to Quantify Adhesive Strength, Air Transport Association Nondestructive Testing Forum, Forth Worth (USA) 17-19.10.2006.

[2] K. Dragan, S. Klimaszewski, Holistic Approach for Structural Integrity Evaluation of Composite Main Rotor Blades, WCNDT Shanghai, China 2008.

[3] D. Findeis, The feasibility of Optical Interference - based NDE Methods to Inspect Helicopter Rotor Blades, Dept. of Mechanical Eng. Univ. of Cape Town 1999.

[4] R. Capriotti, NDT Techniques for the Evaluation of Impact Damage on Aeronautical Structures, Aeronautica Militare Italiana, WCNDT Roma, Italy 2000.

[5] K. Dragan, S. Klimaszewski, A. Leski, Holistic Approach For Assuring Aircraft Availability In Polish Armed Forces, NATO RTO AVT-157 Symposium Ensured Military Platform Availability, 13-16 Montreal, Canada October 2008.

[6] R.R. Adams, P. Cawley, A Review of Defect Types and Non - Destructive Testing Techniques for Composite and Bonded Joints, NDT International, Vol. 21, 1988, p. 208.

[7] Y. Bar-Cohen, In-Service NDE of Aerospace Structures - Emerging Technologies and Challenges at the End of the 2nd Millennium, NDT.net, Vol. 4, September 1999.

[8] R. Smith, Ultrasonic Inspection of Composites, Imperial College of London The Composites Centre, Non Destructive Evaluation of Composite Materials, 9-11 November 2004.
[9] T.C. Miller, B. Lasse, B. VanderHeiden, Composite Damage Detection Using A Novel Ultrasonic Metod, Final Report AFRL-PR-ED-TP-2003-2003-015, Air Force Research Laboratory, Edwards, USA 2003.

[10] K.F. Graff, Waves motion in elastic solids, Dover Publications, Inc., New York 1991.

[11] W. Michnowski, Tłumienie fal ultradźwiękowych, Krajowa Konferencja Badań Nieniszczacych, (Poland) Szczyrk 2001.

[12] http://www.ndt-ed.org/EducationResources/ CommunityCollege/Ultrasonics/Physics/ signaltonoise.htm .

[13] R. Brown, Hanbook of polymer Testing, CRC 1st edition, 1999 .

[14] O. Roach, Enhanced Inspection Methods to Characterize Bonded Joints: Moving Beyond Flaw Detection to Quantify Adhesive Strength, the 49th Annual ATA NDT Forum, Fort Worth, Texas (USA) 16-19 October 2006 .

[15] H. Gundtoft, Quantitative material characterisation of composites by ultrasonic scanning, 15th WCNDT Roma, (Italy) 15-21 October 2000.

[16] K. Dragan, S. Klysz, Detection of damages from the low energy impact damage in the composite structures, I Congress of Polish Mechanics, Warszawa (Poland) August 2007.

[17] R.A. Smith, An introduction to the ultrasonic inspection of composites Non Destructive Evaluation of Composite Materials, Farnborough, November 2004.

[18] X.P.V. Maldague, Theory and practice of infrared technology for non-destructive testing John Wiley\&Sons, Inc. New York 2001.

[19] W. Swiderski, M. Miszczak, Detection of Defects in Composite Pyrotechnic Materials and Products by IR NDT Methods WCNDT Shanghai (China) 2008.

[20] J.P. Fulton, M. Namkung, L.D. Melvin, Practical Estimates of the Errors Associated with the Governing Shearography Equation, Review of Progress in Quantative Nondestructive Equation, La Jolla, California (USA) July 1992, p. 427. 